\title{
EFFECT OF PARAMETRIC RESONANCES ON THE BUNCHED-BEAM DILUTION MECHANISM
}

\author{
M. Ball, B. Brabson, J. Budnick, G. East, B. Hamilton, W.P. Jones, \\ X. Kang, S.Y. Lee, A. Pei, A. Riabko, and T. Sloan \\ Indiana University Cyclotron Facility, Bloomington, Indiana 47408 \\ K.Y. Ng \\ Fermilab, P.O. Box 500, Batavia, Illinois 60510 \\ A.W. Chao, M.G. Minty, and Y. Yan \\ Stanford Linear Accelerator Center, Stanford, California 94309 \\ M. Syphers \\ Brookhaven National Laboratory, Upton, New York 11973
}

Controlled beam-bunch dilution has been proved to be essential in the acceleration of high intensity beams in many high energy accelerators such as the AGS and the CERN PS for transition energy crossing. A secondary RF cavity operating at $200 \mathrm{MHz}$ has been used routinely to blow up the longitudinal phase space for the transition energy crossing. In our recent experiments, we have observed a non-diffusive mechanism in the beam dilution process. As part of a new proposal, we will devote more effort to understand the importance of parametric resonances in bunched-beam dilution.

Double RF systems were used in accelerator physics to decrease space-charge effects by reducing the peak current or to overcome multi-bunch instability by modifying the time structure of the beam. Double RF systems were also found to be a very effective tool for increasing synchrotron tune spread and enchancing the Landau damping rate. In a series of Cooler experiments, we investigated a possibile use of double RF systems in order to stimulate longitudinal beam dilution by modulating the secondary high frequency $R F$ cavity. The experiment described in this paper was motivated by recent theoretical and experimental studies in the field.

This progress report discusses experimental measurements of the evolution of the bunch profile while a modulating secondary RF system is acting on the beam. The experiment was conducted at IUCF and used a bunched proton beam with a kinetic energy of $45 \mathrm{MeV}$. The revolution frequency for the synchronous particle was $f_{0}=1.03168 \mathrm{MHz}$. The frequency of the primary RF cavity was $1.03168 \mathrm{MHz}$ with $h_{1}=1$, and the harmonic number of the secondary rf cavity was $h=9$. The voltage of the main RF cavity was set at about $300 \mathrm{~V}$, which resulted in a synchrotron frequency of about $705 \mathrm{~Hz}$ while operating with the primary $\mathrm{RF}$ cavity alone. The total beam current was about $100 \mu \mathrm{A}$, or equivalently $6 \times 10^{8}$ protons per bunch. The accelerator was operated with a cycle time of $10 \mathrm{~s}$; the injected beam was electron-cooled for about $3 \mathrm{~s}$ (the rms equilibrium bunch length was about $10-20 \mathrm{~ns}$ ); then the phase modulation was applied and beam profiles were digitized for a few hundred milliseconds. To measure the time evolution of the bunch, we used a BPM sum signal passing through a low loss cable $\left(7 / 8^{\prime \prime}\right.$ heliax high bandwidth, low attenuation cable). Then the signal was amplified by an amplifier with a bandwidth of $150 \mathrm{MHz}$ and digitized by a fast sampling scope at a time step of $1 \mathrm{~ns}$. The system 
was controlled by a PC and the data were transferred to a PC via a GPIB control card. A data-processing and replay system has been developed in the $\mathrm{X}$ Window environment. The digitized data can be replayed as a series of frames like a movie, can be integrated to estimate beam loss, or used to compute rms beam size as function of time.

The Hamiltonian for a double RF system with harmonic phase modulation of a high frequency secondary cavity is given by

$$
H=\frac{\nu_{s} \delta^{2}}{2}+\nu_{s}\left((1-\cos \phi)-\frac{r}{h}(1-\cos (h \phi+\Delta \phi(\theta)))\right),
$$

where $\nu_{s}=\left(\frac{h_{1} e V_{1}|\eta|}{2 \pi \beta^{2} E}\right)^{1 / 2}$ is the small amplitude synchrotron tune; $r=\frac{V_{2}}{V_{1}}$ is the ratio of the RF voltages ( $r=0.1,0.15,0.2$ were used in experiment); $h=9$ is the harmonic ratio; $(\phi, \delta)$ are the phases of the particle relative to that of the synchronous particle and the normalized off-momentum variable; $\delta=-\frac{h|\eta|}{\nu_{s}} \frac{\Delta p}{p_{0}}$, where $\eta=-0.86$ is the phase-slip factor; $\Delta \phi(\theta)=A \sin \nu_{m} \theta+\phi_{0}$ is the modulated phase of the secondary RF cavity.

Controlled longitudinal-beam blow up, observed in an experiment, was found to be a very complex process with a very rich and complicated transitional behavior (that fact can be illustrated by a series of beam profiles vs. time). In order to describe this complex phenomenon, we used the following quantities, which are believed to represent the most essential features of the process in question: the rms beam size as function of time $\sigma(t)^{2}=$ $\oint \rho\left(t^{\prime}\right)\left(t-t_{0}\right)^{2} d t^{\prime}$, the saturated value of the rms beam size $\sigma_{m a x}^{2}\left(A, \nu_{m}, \phi_{0}\right)$ and the growth rate $G\left(A, \nu_{m}, \phi_{0}\right)$. After modulation was applied, the beam profile demonstrated diffusive behavior; the rms beam size $\sigma_{t}^{2}(t)$ increased with time and saturated on the time scale of 10-20 synchrotron periods. So, the saturation limit for $\mathrm{rms}$ beam size $\sigma_{t}^{2}$ and the growth rate $G$ are respectively defined as

$$
\sigma_{t}^{2}(t)_{t \rightarrow \infty} \rightarrow \sigma_{m a x}^{2}\left(A, \nu_{m}, \phi_{0}\right) ; \quad G\left(A, \nu_{m}, \phi_{0}\right)=\lim _{t \rightarrow 0} \frac{\sigma_{t}^{2}(t)}{t}
$$

Resonances play an important role in beam-dilution mechanisms. The presence of stable Hamiltonian tori determines the limits on beam growth. A derivation, presented below, shows how a system of resonances can be derived for a Hamiltonian. ${ }^{1}$ It is possible to divide the original Hamiltonian into a sum of the time-independent Hamiltonian and the time-dependent perturbation as $H=H_{0}+\Delta H(t)$. We find from numerical simulations that the diffusion rate depends sensitively on the phase difference between the main and secondary RF cavities, $\phi_{0}$. A phase shift of $180^{\circ}$ flattens the total RF potential near the origin; that makes diffusion stronger. In order to achieve a good agreement between numerical simulations and experimental data, it is necessary to include the combined effect of noise and damping (it is especially important for high modulation frequencies). Figure 1 compares the rms beam width obtained from numerical simulations with those obtained from experimental measurements.

In conclusion, we have observed controlled longitudinal-beam blow up when the phase of a secondary high-requency RF cavity was modulated. Different amplitudes and frequencies for the external modulation were used in this experiment. It was confirmed that resonances played an important role in the beam-dilution mechanism. This fact was 

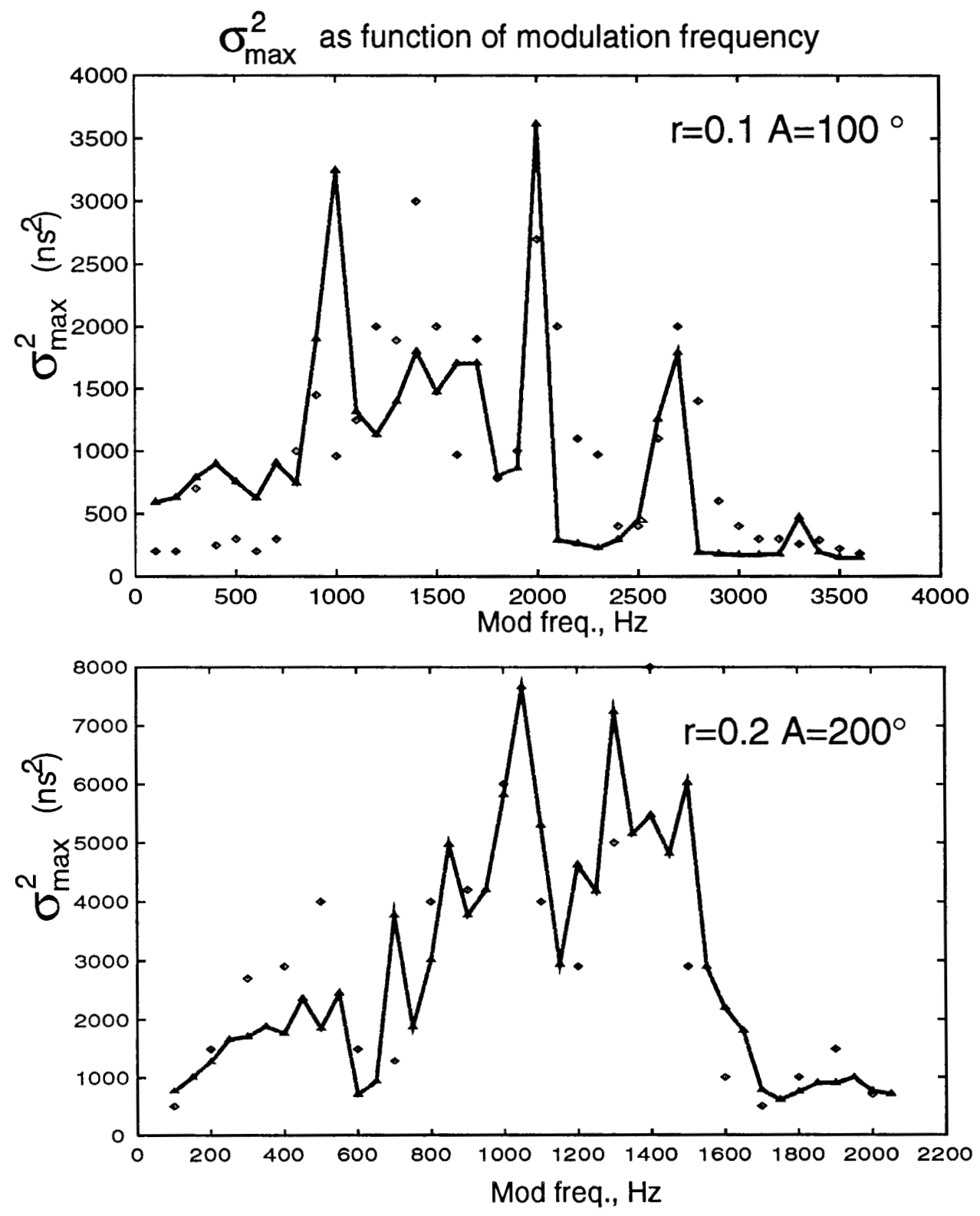

Figure 1. The solid line shows the results of numerical simulations and the dots are derived from experimental data for a) $r=0.1 A=100^{\circ}$ shown in the upper plot and b) $r=0.2 A=200^{\circ}$ shown in the lower plot.

qualitively explained by the methods of nonlinear Hamiltonian dynamics. Numerical simulations were performed and found to be in qualitative agreement with the experimental data. It was also established that the diffusion process strongly depends on the phase difference between the main and secondary RF cavities. 